\title{
On the notions of suborbifold and orbifold embedding
}

\author{
JOSEPH E BORZELLINO \\ VICTOR BRUNSDEN
}

\begin{abstract}
The purpose of this article is to investigate the relationship between suborbifolds and orbifold embeddings. In particular, we give natural definitions of the notion of suborbifold and orbifold embedding and provide many examples. Surprisingly, we show that there are (topologically embedded) smooth suborbifolds which do not arise as the image of a smooth orbifold embedding. We are also able to characterize those suborbifolds that can arise as the images of orbifold embeddings. As an application, we show that a length-minimizing curve (a geodesic segment) in a Riemannian orbifold can always be realized as the image of an orbifold embedding.
\end{abstract}

57R18; 57R35, 57R40

\section{Introduction}

The purpose of this article is to investigate some of the difficulties and subtleties associated with the study of the differential topology of smooth orbifolds. It will be no surprise to anyone who has taken more than a cursory look at orbifolds that the goal of extending the most basic notions from the differential topology of manifolds to orbifolds has not been achieved in a universally accepted manner in the nearly 60 years since Satake $[17 ; 18]$ introduced $V$-manifolds (now orbifolds as popularized by Thurston [19]). In the six decades since they were introduced, there has been a proliferation of definitions and ad hoc refinements each used to overcome some inherent difficulty unearthed while attempting an orbifold generalization of a manifold result. These challenges are readily acknowledged by experts and often provide the inspiration for new research on orbifolds. In fact, it has been humorously mentioned that there exists today a partial ordering for the plethora of definitions related to orbifolds, and that one can only imagine what an application of Zorn's lemma might yield! The aim here is much less ambitious. Our goal is to expose and investigate in detail the subtle notion of suborbifold and its relation to the natural idea of an orbifold embedding. Some of the particular difficulties involving the notion of suborbifolds and orbifold embeddings have already been noted in the orbifold literature; see Borzellino and Brunsden [4; 5; 6], Chen and Ruan [9] and Adem, Leida and Ruan [1], and more recently Lerman [14], 
Choi [11] and Cho, Hong and Shin [10]. For manifolds, it is a fundamental result of differential topology that submanifolds are precisely the images of embeddings; see Hirsch [12, Theorem 3.1]. In fact, many authors use this characterization as the definition of submanifold. Our main result identifies necessary and sufficient conditions which characterize precisely when a suborbifold can be realized as the image of an orbifold embedding. Unlike the case for manifolds, we also show that suborbifolds exist which are not the images of orbifold embeddings.

Theorem 1 Let $\mathcal{P}$ be a smooth suborbifold of a smooth orbifold $\mathcal{O}$.

(1) There exists an orbifold $\mathcal{P}^{\prime}$ and a topological embedding of underlying spaces $\iota: X_{\mathcal{P}^{\prime}} \rightarrow X_{\mathcal{O}}$ so that $\iota\left(X_{\mathcal{P}^{\prime}}\right)=X_{\mathcal{P}}$ if and only if $\mathcal{P}$ is saturated.

(2) There exists a complete orbifold embedding $\star_{\star} \iota=\left(\iota,\left\{\tilde{i}_{x}\right\},\left\{\Theta_{\iota, x}\right\}\right): \mathcal{P}^{\prime} \rightarrow \mathcal{O}$ covering $\iota$ if and only if $\mathcal{P}$ is both saturated and split.

The definitions of what it means for a suborbifold to be saturated or split appear in Section 2.1. The definition of complete orbifold map appears in Section 3.1.

As an application of Theorem 1 to length-minimizing geodesics in Riemannian orbifolds, we have the following corollary which follows from the characterization of lengthminimizing geodesic segments found in Borzellino [2; 3].

Corollary 2 Let $\mathcal{O}$ be a Riemannian orbifold and let $X \subset X_{\mathcal{O}}$ be the underlying point set of a length-minimizing curve joining two points of $\mathcal{O}$. Then there is a suborbifold $\mathcal{P} \subset \mathcal{O}$ whose underlying space $X_{\mathcal{P}}=X$ is the image of a complete orbifold embedding.

\section{Orbifold background}

Although there are many references for this background material, we will use our previous work $[5 ; 7]$ as our standard reference. While much of what we discuss here works equally well for smooth $C^{r}$ orbifolds, to simplify the exposition, we restrict ourselves to smooth $C^{\infty}$ orbifolds. Throughout, the term smooth means $C^{\infty}$. This results in no loss of generality [5, Proposition $3.11 ; 13]$. Note that the classical definition of orbifold given below is modeled on the definition in Thurston [19] and that these orbifolds are referred to as classical effective orbifolds in [1].

Definition 3 An $n$-dimensional smooth orbifold $\mathcal{O}$, consists of a paracompact, Hausdorff topological space $X_{\mathcal{O}}$, called the underlying space, with the following local structure. For each $x \in X_{\mathcal{O}}$ and neighborhood $U$ of $x$, there is a neighborhood $U_{x} \subset U$, 
an open set $\tilde{U}_{x}$ diffeomorphic to $\mathbb{R}^{n}$, a finite group $\Gamma_{x}$ acting smoothly and effectively on $\tilde{U}_{x}$ which fixes $0 \in \tilde{U}_{x}$, and a homeomorphism $\phi_{x}: \tilde{U}_{x} / \Gamma_{x} \rightarrow U_{x}$ with $\phi_{x}(0)=x$. These actions are subject to the condition that for a neighborhood $U_{z} \subset U_{x}$ with corresponding $\tilde{U}_{z} \cong \mathbb{R}^{n}$, group $\Gamma_{z}$ and homeomorphism $\phi_{z}: \tilde{U}_{z} / \Gamma_{z} \rightarrow U_{z}$, there is a smooth embedding $\widetilde{\psi}_{z x}: \tilde{U}_{z} \rightarrow \widetilde{U}_{x}$ and an injective homomorphism $\theta_{z x}: \Gamma_{z} \rightarrow \Gamma_{x}$ so that $\tilde{\psi}_{z x}$ is equivariant with respect to $\theta_{z x}$ (that is, for $\gamma \in \Gamma_{z}, \tilde{\psi}_{z x}(\gamma \cdot \tilde{y})=\theta_{z x}(\gamma) \cdot \tilde{\psi}_{z x}(\tilde{y})$ for all $\tilde{y} \in \tilde{U}_{z}$ ), such that the following diagram commutes:

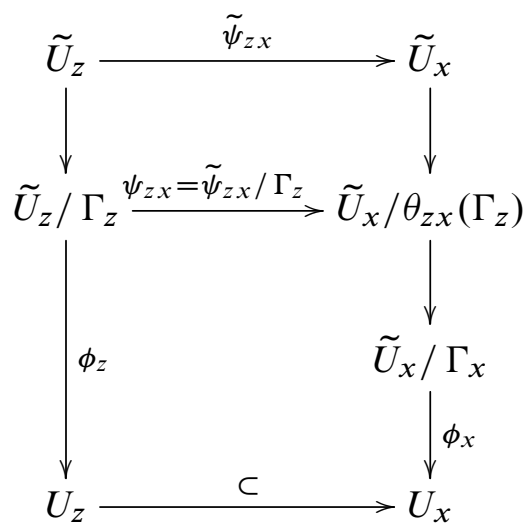

We will refer to the neighborhood $U_{x}$ or $\left(\tilde{U}_{x}, \Gamma_{x}\right)$ or $\left(\tilde{U}_{x}, \Gamma_{x}, \rho_{x}, \phi_{x}\right)$ as an orbifold chart, and write $U_{x}=\widetilde{U}_{x} / \Gamma_{x}$. In the 4-tuple notation, we are making explicit the representation $\rho_{x}: \Gamma_{x} \rightarrow \operatorname{Diff}^{\infty}\left(\tilde{U}_{x}\right)$. The isotropy group of $x$ is the group $\Gamma_{x}$. The definition of orbifold implies that the germ of the action of $\Gamma_{x}$ in a neighborhood of the origin of $\mathbb{R}^{n}$ is unique, so that by shrinking $\tilde{U}_{x}$ if necessary, $\Gamma_{x}$ is well-defined up to isomorphism. The singular set of $\mathcal{O}$ is the set of points $x \in \mathcal{O}$ with $\Gamma_{x} \neq\{e\}$. More detail can be found in [5].

\subsection{Smooth suborbifolds}

Originally, in [19], the notion of an $m$-suborbifold $\mathcal{P}$ of an $n$-orbifold $\mathcal{O}$ required $\mathcal{P}$ to be locally modeled on $\mathbb{R}^{m} \subset \mathbb{R}^{n}$ modulo finite groups. That is, the local action on $\mathbb{R}^{m}$ is induced by the local action on $\mathbb{R}^{n}$. As interest in the differential topology of orbifolds grew, it was discovered early, for instance in [4], that this definition was too restrictive to admit, for example, the diagonal embedding of an orbifold as a suborbifold of the product orbifold. Other authors $[1 ; 9 ; 10]$ overcame this difficulty by defining their suborbifolds explicitly as images of their particular notion of orbifold embedding in analogy with the case of manifolds. In [6], we defined a notion of suborbifold which is general enough to include the diagonal embedding as a suborbifold of the product, but is independent of our notion of orbifold embedding which we recall in Section 3. 
Using our definition of suborbifold we can also easily identify those suborbifolds in the original sense of Thurston [19]. We refer to them as full suborbifolds. Recall the definition of suborbifold from [6]:

Definition 4 An (embedded) suborbifold $\mathcal{P}$ of an orbifold $\mathcal{O}$ consists of the following:

(1) A subspace $X_{\mathcal{P}} \subset X_{\mathcal{O}}$ equipped with the subspace topology

(2) For each $x \in X_{\mathcal{P}}$ and neighborhood $W$ of $x$ in $X_{\mathcal{O}}$ there is an orbifold chart $\left(\tilde{U}_{x}, \Gamma_{x}, \rho_{x}, \phi_{x}\right)$ about $x$ in $\mathcal{O}$ with $U_{x} \subset W$, a subgroup $\Lambda_{x} \subset \Gamma_{x}$ of the isotropy group of $x$ in $\mathcal{O}$ and a $\rho_{x}\left(\Lambda_{x}\right)$ invariant submanifold $\tilde{V}_{x} \subset \tilde{U}_{x} \cong \mathbb{R}^{n}$, so that $\left(\widetilde{V}_{x}, \Lambda_{x} / \Omega_{x},\left.\rho_{x}\right|_{\Lambda_{x}}, \psi_{x}\right)$ is an orbifold chart for $\mathcal{P}$, where $\Omega_{x}=\{\gamma \in$ $\left.\Lambda_{x}:\left.\rho_{x}(\gamma)\right|_{\tilde{V}_{x}}=\operatorname{Id}\right\}$. (In particular, the intrinsic isotropy subgroup at $x \in \mathcal{P}$ is $\left.\Lambda_{x} / \Omega_{x}\right)$.

(3) For each $x$ in $\mathcal{P}, V_{x}=\psi_{x}\left(\tilde{V}_{x} / \rho_{x}\left(\Lambda_{x}\right)\right)=U_{x} \cap X_{\mathcal{P}}$ is an orbifold chart.

Implicit in this definition is the requirement that the invariant submanifolds $\tilde{V}_{x}$ be smooth, and that the collection of charts $\left\{\left(\widetilde{V}_{x}, \Lambda_{x} / \Omega_{x},\left.\rho_{x}\right|_{\Lambda_{x}}, \psi_{x}\right)\right\}$ satisfy the compatibility conditions of Definition 3 , thus giving $\mathcal{P}$ the structure of a smooth orbifold. Condition (2) of this definition is not very restrictive as we shall see later in this section. Thurston's notion of suborbifold [19] is equivalent to adding the condition that $\Lambda_{x}=\Gamma_{x}$ at all $x$ in the underlying topological space of $\mathcal{P}$, and so we make the following definition:

Definition $5 \mathcal{P} \subset \mathcal{O}$ is a full suborbifold of $\mathcal{O}$ if $\mathcal{P}$ is a suborbifold with $\Lambda_{x}=\Gamma_{x}$ for all $x \in \mathcal{P}$.

When necessary for clarity, we will use the notation $\Gamma_{x, \mathcal{O}}$ to denote the intrinsic isotropy of a point $x$ in an orbifold $\mathcal{O}$, and use the subscript $\mathcal{O}$ as well on needed subgroups of $\Gamma_{x, \mathcal{O}}$. Observe that in the case of a suborbifold $\mathcal{P} \subset \mathcal{O}$ we always have the exact sequence of groups

$$
1 \longrightarrow \Omega_{x, \mathcal{O}} \longrightarrow \Lambda_{x, \mathcal{O}} \subset \Gamma_{x, \mathcal{O}} \longrightarrow \Gamma_{x, \mathcal{P}} \longrightarrow 1,
$$

where $\Gamma_{x, \mathcal{P}}$ denotes the intrinsic isotropy group of $\mathcal{P}$ at $x$.

In characterizing those suborbifolds that are images of orbifold embeddings, we need the following two definitions. 
Definition 6 We say that $\mathcal{P} \subset \mathcal{O}$ is a split suborbifold of $\mathcal{O}$ if the exact sequence above is (right) split for all $x \in \mathcal{P}$. That is, there is a group homomorphism $\sigma: \Gamma_{x, \mathcal{P}} \rightarrow$ $\Lambda_{x, \mathcal{O}}$ such that the composition $q \circ \sigma=\mathrm{Id}$, where $q: \Lambda_{x, \mathcal{O}} \rightarrow \Gamma_{x, \mathcal{P}}$ is the quotient homomorphism:

$$
1 \longrightarrow \Omega_{x, \mathcal{O}} \longrightarrow \Lambda_{x, \mathcal{O}} \underset{\sigma}{\stackrel{q}{\rightleftarrows}} \Gamma_{x, \mathcal{P}} \longrightarrow 1 .
$$

Note that if $\mathcal{P} \subset \mathcal{O}$ is split, we have $\Lambda_{x, \mathcal{O}} \cong \Omega_{x, \mathcal{O}} \rtimes \Gamma_{x, \mathcal{P}}$, a semidirect product, and in the case that the groups are abelian $\Lambda_{x, \mathcal{O}} \cong \Omega_{x, \mathcal{O}} \times \Gamma_{x, \mathcal{P}}$, the direct product. Of course, if $\Omega_{x, \mathcal{O}}$ or $\Gamma_{x, \mathcal{P}}$ is trivial, then $\mathcal{P}$ is split as well.

Definition 7 We say that $\mathcal{P} \subset \mathcal{O}$ is a saturated suborbifold of $\mathcal{O}$ if for each $x \in \mathcal{P}$ and $\tilde{y} \in \tilde{V}_{x}$, we have that $\left(\Gamma_{x, \mathcal{O}} \cdot \tilde{y}\right) \cap \tilde{V}_{x}=\Lambda_{x, \mathcal{O}} \cdot \tilde{y}$.

The saturation condition can be thought of as a kind of orbit maximality condition on the group $\Lambda_{x, \mathcal{O}} \subset \Gamma_{x, \mathcal{O}}$ relative to the invariant submanifold $\tilde{V}_{x}$. Observe that, by definition, every full suborbifold is automatically saturated.

Example 8 Let $\mathcal{Q}=\mathbb{R} / \mathbb{Z}_{2}$ be the smooth orbifold (without boundary), where $\mathbb{Z}_{2}$ acts on $\mathbb{R}$ via $\gamma \cdot x=-x$. The underlying topological space $X_{\mathcal{Q}}$ of $\mathcal{Q}$ is $[0, \infty)$ and the isotropy subgroups are $\{e\}$ for $x \in(0, \infty)$ and $\mathbb{Z}_{2}$ for $x=0$. Let $\mathcal{O}=\mathcal{Q} \times \mathcal{Q}$ be the smooth product orbifold (without boundary). See [5, Definition 2.12]. The underlying space for $\mathcal{O}$ can be identified with the closed first quadrant and the singular points of $\mathcal{O}$ lie in one of three connected singular strata: the positive $x$ axis, the positive $y$ axis (corresponding to those points with $\mathbb{Z}_{2}$ isotropy), and the origin which has $\mathbb{Z}_{2} \times \mathbb{Z}_{2}$ isotropy. Then $\mathcal{P}=\{0\} \times \mathcal{Q}$ is a full (and thus, saturated) suborbifold of $\mathcal{O}$. To see this, note that $\Gamma_{(0,0), \mathcal{P}} \cong \mathbb{Z}_{2}, \Gamma_{(0,0), \mathcal{O}} \cong \mathbb{Z}_{2} \times \mathbb{Z}_{2}$, and that $\Omega_{(0,0), \mathcal{O}}=\left\{\gamma \in \Gamma_{(0,0), \mathcal{O}}\right.$ : $\left.\left.\gamma\right|_{\{0\} \times \mathbb{R}}=\mathrm{Id}\right\} \cong \mathbb{Z}_{2}$. Thus, $\Gamma_{(0,0), \mathcal{P}} \cong \Gamma_{(0,0), \mathcal{O}} / \Omega_{(0,0), \mathcal{O}}$. Similarly, $\mathcal{P}=\mathcal{Q} \times\{0\}$ is a full suborbifold. Each of these suborbifolds is split as well. See Figure 1.
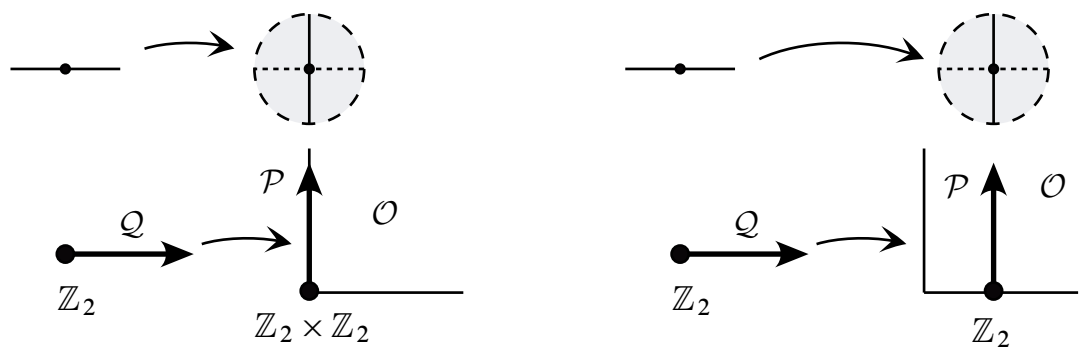

Figure 1: Examples 8 and 9 
Example 9 Let $\mathcal{Q}$ and $\mathcal{O}$ be as in Example 8. Then $\mathcal{P}=\{1\} \times \mathcal{Q}$ is a full (thus, saturated), split suborbifold of $\mathcal{O}$. In this case, note that $\Gamma_{(1,0), \mathcal{P}} \cong \mathbb{Z}_{2}, \Gamma_{(1,0), \mathcal{O}} \cong$ $\mathbb{Z}_{2}$, and that $\Omega_{(1,0), \mathcal{O}}=\left\{\gamma \in \Gamma_{(1,0), \mathcal{O}}:\left.\gamma\right|_{\{1\} \times \mathbb{R}}=\operatorname{Id}\right\}=\{e\}$. Thus, $\Gamma_{(1,0), \mathcal{P}} \cong$ $\Gamma_{(1,0), \mathcal{O}} / \Omega_{(1,0), \mathcal{O}}$. See Figure 1 .

Example 10 [5, Example 2.15] Let $\mathcal{Q}$ and $\mathcal{O}$ be as in Example 8. Then the diagonal $\mathcal{P}=\operatorname{diag}(\mathcal{Q})=\{(x, x): x \in \mathcal{Q}\} \subset \mathcal{O}$ is a suborbifold. Here, $\Gamma_{(0,0), \mathcal{P}} \cong \mathbb{Z}_{2}, \Gamma_{(0,0), \mathcal{O}} \cong$ $\mathbb{Z}_{2} \times \mathbb{Z}_{2}$, and $\Omega_{(0,0), \mathcal{O}}=\left\{\gamma \in \Gamma_{(0,0), \mathcal{O}}:\left.\gamma\right|_{\operatorname{diag}(\mathbb{R}) \subset \mathbb{R}^{2}}=\operatorname{Id}\right\}=\{e\}$. Note that $\Gamma_{(0,0), \mathcal{P}} \not$ $\Gamma_{(0,0), \mathcal{O}} / \Omega_{(0,0), \mathcal{O}}$. Thus, $\mathcal{P}$ is not a full suborbifold. However, $\mathcal{P}$ is split and saturated since $\Gamma_{(0,0), \mathcal{P}} \cong \Lambda_{(0,0), \mathcal{O}} / \Omega_{(0,0), \mathcal{O}}$, where $\mathbb{Z}_{2} \cong \Lambda_{(0,0), \mathcal{O}} \subset \Gamma_{(0,0), \mathcal{O}}$ is the diagonal embedding of $\mathbb{Z}_{2} \hookrightarrow \mathbb{Z}_{2} \times \mathbb{Z}_{2}$ given by $\gamma \mapsto(\gamma, \gamma)$. See Figure 2 .
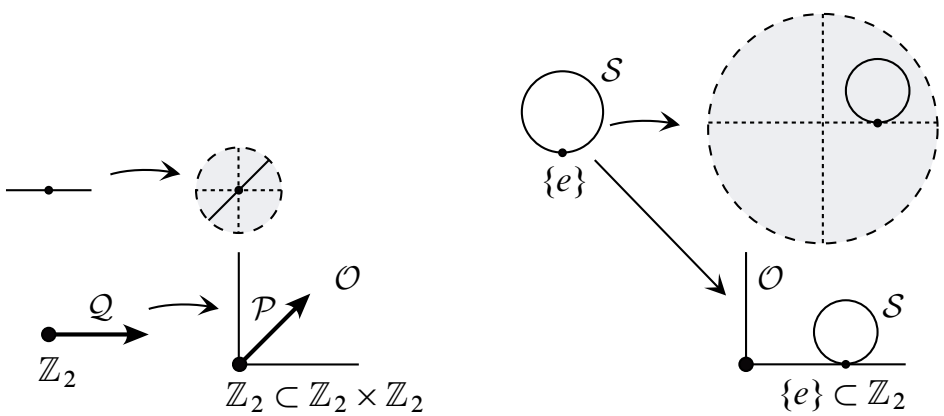

Figure 2: Examples 10 and 11

Example 11 Let $\mathcal{O}$ be as in Example 8. Consider the circle $\mathcal{S} \subset \mathcal{O}$ of radius 1 centered at $(2,1)$. Then $\mathcal{S}$ is a suborbifold of $\mathcal{O}$ that is not a full suborbifold. To see this, just note that at the point $x=(2,0) \in \mathcal{O}$ any lift of $\mathcal{S}$ to $\tilde{U}_{x} \cong \mathbb{R}^{2}$ in a neighborhood of $x$, cannot be an invariant submanifold unless we choose $\Lambda_{x, \mathcal{O}}=\{e\}$. In this case, we see that the intrinsic isotropy group of $\mathcal{S}$ at $x$ is trivial which it must be since $\mathcal{S}$ is actually a compact 1 -dimensional manifold. That is, a compact 1 -dimensional orbifold with trivial orbifold structure. It is easy to see that $\mathcal{S}$ is saturated and split as well. See Figure 2.

Each of the previous examples will be seen to be the image of an orbifold embedding in Section 3.2. However, the following three examples of suborbifolds will be shown not to be the image of an orbifold embedding.

Example 12 Let $\mathcal{O}=\mathbb{C}^{2} / \mathbb{Z}_{4}$, where $\mathbb{Z}_{4}$ acts on $\mathbb{C}^{2}$ via the matrix group

$$
\mathbb{Z}_{4} \cong\left\{\left(\begin{array}{cc}
i^{k} & 0 \\
0 & (-1)^{k}
\end{array}\right): k \in\{0,1,2,3\}\right\} \text {. }
$$


Then $\mathcal{P}=(\{0\} \times \mathbb{C}) / \mathbb{Z}_{4} \cong \mathbb{C} / \mathbb{Z}_{2}$ is a full, (hence, saturated) suborbifold of $\mathcal{O}$. In this case, for $x=(0,0)$, we have $\Omega_{x, \mathcal{O}} \cong \mathbb{Z}_{2}, \Lambda_{x, \mathcal{O}}=\Gamma_{x, \mathcal{O}} \cong \mathbb{Z}_{4}$, and $\Gamma_{x, \mathcal{P}} \cong \mathbb{Z}_{2}$. Hence, the corresponding suborbifold exact sequence for $\mathcal{P}$ is $1 \rightarrow \mathbb{Z}_{2} \rightarrow \mathbb{Z}_{4} \rightarrow \mathbb{Z}_{2} \rightarrow 1$ which is clearly not split.

The next example illustrates how flexible the seemingly straightforward definition of suborbifold actually is.

Example 13 Consider the 2-dimensional orbifolds $\mathcal{O}_{1}=\mathbb{C}=\mathbb{C} / \mathbb{Z}_{1}, \mathcal{O}_{2}=\mathbb{C} / \mathbb{Z}_{2}$, $\mathcal{O}_{3}=\mathbb{C} / \mathbb{Z}_{4}$, and $\mathcal{O}_{4}=\mathbb{C} / \mathbb{Z}_{8}$. Here $\mathbb{Z}_{k}$ acts on $\mathbb{C}$ via multiplication by $e^{2 \pi i / k}$, $z \mapsto e^{2 \pi i / k} z$. According to Definition 4, we have $\mathcal{O}_{1} \subset \mathcal{O}_{2} \subset \mathcal{O}_{3} \subset \mathcal{O}_{4}$ as suborbifolds. The underlying topological spaces $X_{\mathcal{O}_{n}}$ are all (topologically) homeomorphic, to a standard cone over a circle. It is easily checked that none of these is a saturated suborbifold of one of the others and since $\Omega_{0, \mathcal{O}_{n}}=\{e\}, \mathcal{O}_{m} \subset \mathcal{O}_{n},(m<n)$ are all split suborbifolds.

Example 14 Let $\mathcal{O}$ and $\mathcal{P}$ be as in Example 12. Let $\mathcal{Q}=\operatorname{diag}(\mathcal{P})=\{(x, x) \in \mathcal{O} \times \mathcal{O}$ : $x \in \mathcal{P}\}$. Let $\mathcal{R}=\mathcal{O} \times \mathcal{O}$. Let $x=(0,0)$. Then $\Gamma_{x, \mathcal{R}} \cong \mathbb{Z}_{4} \times \mathbb{Z}_{4}$. Analogous to Example 10 , we see that $\Gamma_{x, \mathcal{Q}} \cong \mathbb{Z}_{2}, \Lambda_{x, \mathcal{R}} \cong \mathbb{Z}_{4}$, and $\Omega_{x, \mathcal{R}} \cong \mathbb{Z}_{2}$. Thus, $\mathcal{Q}$ is not split in $\mathcal{R}$ and is not a full suborbifold of $\mathcal{R}$. On the other hand, it is not hard to see that $\mathcal{Q}$ is saturated in $\mathcal{R}$.

Our last example shows that even though the underlying space of a smooth orbifold (without boundary) may be topologically embedded as a subspace of the underlying space of another smooth orbifold, this subspace (with its independent orbifold structure) may not be a suborbifold of the ambient orbifold.

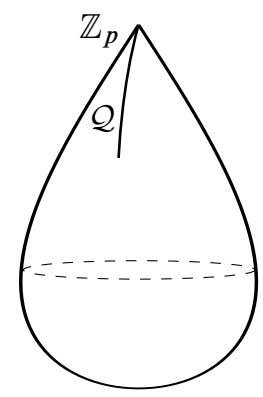

Figure 3: Example 15

Example 15 Let $\mathcal{O}$ be a so-called $\mathbb{Z}_{p}$-teardrop, and let $\mathcal{Q}=\mathbb{R} / \mathbb{Z}_{2}$ be as in Example 8, a smooth one-dimensional orbifold (without boundary). Let $X_{\mathcal{Q}} \subset X_{\mathcal{O}}$ be topologically 
embedded as a half-interval starting at the point $x$. See Figure 3. As a nontrivial 1 -orbifold, the intrinsic isotropy group for $\mathcal{Q}$ at $x$ must be $\mathbb{Z}_{2}$. Thus the order of the ambient isotropy group $\Gamma_{x, \mathcal{O}}$ must be even. We conclude that $\mathcal{Q}$ is not a suborbifold of $\mathcal{O}$ when $p$ is odd. Of course, if $p$ is even, then it is possible for $\mathcal{Q}$ to be a suborbifold of $\mathcal{O}$ which is saturated and split and thus an embedded suborbifold.

\section{Smooth mappings between orbifolds}

In the literature, there are four related definitions of maps between orbifolds which are based on the classical Satake-Thurston approach to orbifolds via atlases of orbifold charts. In this paper, we use the notion of complete orbifold map. It is distinguished from the other notions of orbifold map in that it keeps track of all defining data. All other notions of orbifold map descend from the complete orbifold maps by forgetting information. In the special case of embeddings, however, the property of being an embedding passes down from the complete orbifold maps to the level of orbifold maps. This observation requires only an understanding on how these two notions of orbifold map are related to one another. We point this out explicitly in our exposition below. We refer the reader to [7] for the necessary background details and in what follows we use the notation of [5, Section 2].

The original motivation for defining the notion of complete orbifold map was to make meaningful and well-defined certain geometric constructions involving orbifolds and their maps. The need to be careful in defining an adequate notion of orbifold map was already noted in the work of Moerdijk and Pronk [15] and Chen and Ruan [8] and was missing from Satake's original work on $V$-manifolds [17; 18]. More recently, Pohl [16] developed another notion of orbifold morphism to address some inconsistencies in earlier work using the groupoid approach to orbifolds.

\subsection{Mappings between orbifolds}

Definition 16 A $C^{\infty}$ complete orbifold map $\star f=\left(f,\left\{\tilde{f}_{x}\right\},\left\{\Theta_{f, x}\right\}\right)$ between smooth orbifolds $\mathcal{P}$ and $\mathcal{O}$ consists of the following:

(1) A continuous map $f: X_{\mathcal{P}} \rightarrow X_{\mathcal{O}}$ of the underlying topological spaces.

(2) For each $y \in \mathcal{P}$, a group homomorphism $\Theta_{f, y}: \Gamma_{y} \rightarrow \Gamma_{f(y)}$.

(3) A smooth $\Theta_{f, y}$-equivariant lift $\tilde{f}_{y}: \tilde{U}_{y} \rightarrow \tilde{V}_{f(y)}$, where $\left(\tilde{U}_{y}, \Gamma_{y}\right)$ is an orbifold chart at $y$ and $\left(\tilde{V}_{f(y)}, \Gamma_{f(y)}\right)$ is an orbifold chart at $f(y)$. That is, the following diagram commutes: 


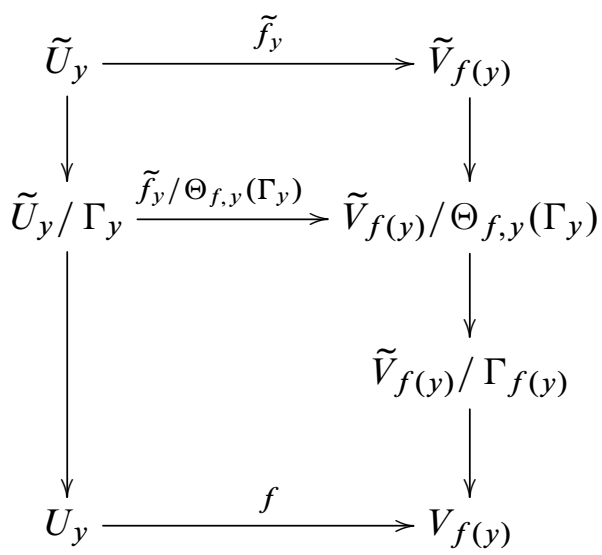

(ネ4) (Equivalence) Two complete orbifold maps

$$
\star f=\left(f,\left\{\tilde{f}_{x}\right\},\left\{\Theta_{f, x}\right\}\right) \text { and } \star g=\left(g,\left\{\widetilde{g}_{x}\right\},\left\{\Theta_{g, x}\right\}\right)
$$

are considered equivalent if for each $x \in \mathcal{P}, \tilde{f}_{x}=\widetilde{g}_{x}$ as germs and $\Theta_{f, x}=\Theta_{g, x}$. That is, there exists an orbifold chart $\left(\widetilde{U}_{x}, \Gamma_{x}\right)$ at $x$ such that $\left.\widetilde{f}_{x}\right|_{\tilde{U}_{x}}=\left.\tilde{g}_{x}\right|_{\tilde{U}_{x}}$ and $\Theta_{f, x}=\Theta_{g, x}$. Note that this implies that $f=g$.

The set of smooth complete orbifold maps from $\mathcal{P}$ to $\mathcal{O}$ will be denoted by $C_{\star \text { Orb }}^{\infty}(\mathcal{P}, \mathcal{O})$. For $\mathcal{P}$ compact (without boundary), $C_{\star \text { Orb }}^{\infty}(\mathcal{P}, \mathcal{O})$ carries the structure of a smooth Fréchet manifold [7].

The following condition can replace $(\star 4)$ in Definition 16 :

(4) (Equivalence) Two complete orbifold maps

$$
\left(f,\left\{\tilde{f}_{x}\right\},\left\{\Theta_{f, x}\right\}\right) \text { and }\left(g,\left\{\tilde{g}_{x}\right\},\left\{\Theta_{g, x}\right\}\right)
$$

are considered equivalent if for each $x \in \mathcal{P}, \tilde{f}_{x}=\widetilde{g}_{x}$ as germs. That is, there exists an orbifold chart $\left(\widetilde{U}_{x}, \Gamma_{x}\right)$ at $x$ such that $\left.\widetilde{f}_{x}\right|_{\tilde{U}_{x}}=\left.\widetilde{g}_{x}\right|_{\tilde{U}_{x}}$ (which as before implies $f=g$ ).

Here we have dropped the requirement that $\Theta_{f, x}=\Theta_{g, x}$. If we do this, we recover the notion of orbifold map $\left(f,\left\{\tilde{f}_{x}\right\}\right)$ which appeared in [5, Section 3]. Thus, the set of orbifold maps $C_{\mathrm{Orb}}^{\infty}(\mathcal{P}, \mathcal{O})$ can be regarded as the equivalence classes of complete orbifold maps under the less restrictive set-theoretic equivalence (4). For $\mathcal{P}$ compact (without boundary), $C_{\mathrm{Orb}}^{\infty}(\mathcal{P}, \mathcal{O})$ carries the structure of a stratified space whose strata are modeled on smooth Fréchet manifolds [7]. 


\subsection{Orbifold embeddings}

Definition 17 A complete orbifold map $\star f=\left(f,\left\{\tilde{f}_{x}\right\},\left\{\Theta_{f, x}\right\}\right)$ between smooth orbifolds $\mathcal{P}$ and $\mathcal{O}$ is a complete orbifold embedding if the map $f: X_{\mathcal{P}} \rightarrow X_{\mathcal{O}}$ is a topological embedding of the underlying spaces, each of the homomorphisms $\Theta_{f, y}: \Gamma_{y} \rightarrow \Gamma_{f(y)}$ is injective and the $\Theta_{f, y}$-equivariant local lifts $\tilde{f}_{y}: \tilde{U}_{y} \rightarrow \widetilde{V}_{f(y)}$ are smooth embeddings on each chart.

One should observe that the condition that the equivariant local lifts $\tilde{f}_{x}$ are embeddings automatically implies that the corresponding homomorphisms $\Theta_{f, x}$ are injective: If there exists $\gamma \in \Gamma_{x}$ with $\Theta_{f, x}(\gamma)=\{e\}$, then equivariance of $\tilde{f}_{x}$ yields $\tilde{f}_{x}(\gamma \cdot \tilde{y})=$ $\Theta_{f, x}(\gamma) \cdot \tilde{f}_{x}(\tilde{y})=\tilde{f}_{x}(\tilde{y})$ for all $\tilde{y} \in \widetilde{U}_{x}$. Since $\tilde{f}_{x}$ is an embedding this implies that $\gamma=\{e\}$, and thus $\Theta_{f, x}$ is injective. Thus the condition that $\Theta_{f, y}$ be injective is redundant for embeddings. As a consequence, there is a sensible definition of orbifold embedding in the category of orbifold maps as well:

Definition 18 An orbifold map $f=\left(f,\left\{\tilde{f}_{x}\right\}\right)$ between smooth orbifolds $\mathcal{P}$ and $\mathcal{O}$ is an orbifold embedding if the map $f: X_{\mathcal{P}} \rightarrow X_{\mathcal{O}}$ is a topological embedding of the underlying spaces, and on each chart, the $\Theta_{f, y}$-equivariant local lifts $\tilde{f}_{y}: \widetilde{U}_{y} \rightarrow \widetilde{V}_{f(y)}$ are smooth embeddings.

The following example from [7, Section 2] is illustrative.

Example 19 Let $\mathcal{Q}=\mathbb{R} / \mathbb{Z}_{2}$ be as in Example 8. Consider the inclusion (embedding) $f: \mathcal{Q} \rightarrow \mathcal{Q} \times \mathcal{Q} \times \mathcal{Q}, y \mapsto(y, 0,0)$, where $\tilde{f}_{x}(\tilde{y})=(\tilde{y}, 0,0)$. Note that $\tilde{f}_{0}$ is equivariant with respect to both $\Theta_{f, 0}(\gamma)=(\gamma, e, e)$ and $\Theta_{f, 0}^{\prime}(\gamma)=(\gamma, \gamma, \gamma)$. Thus, we have two distinct complete orbifold embeddings

$$
\star f=\left(f,\left\{\tilde{f}_{x}\right\},\left\{\Theta_{f, x}\right\}\right) \text { and } \star f^{\prime}=\left(f,\left\{\tilde{f}_{x}\right\},\left\{\Theta_{f, x}^{\prime}\right\}\right)
$$

which represent the same orbifold embedding $f=\left(f,\left\{\tilde{f}_{x}\right\}\right)$. In each case, observe that both $\Theta_{f, x}$ and $\Theta_{f, x}^{\prime}$ are injective confirming the remarks which followed Definition 17.

For open embeddings, that is, in the case where $\operatorname{dim}(\mathcal{P})=\operatorname{dim}(\mathcal{O})$, it is useful to note that the phenomenon in Example 19 cannot occur [7, Section 4]. To see this, note that if two complete orbifold embeddings

$$
\star f=\left(f,\left\{\tilde{f}_{x}\right\},\left\{\Theta_{f, x}\right\}\right) \text { and } \star f^{\prime}=\left(f,\left\{\tilde{f}_{x}\right\},\left\{\Theta_{f, x}^{\prime}\right\}\right)
$$

represent the same orbifold embedding $f=\left(f,\left\{\tilde{f}_{x}\right\}\right)$, then equivariance of $\tilde{f}_{x}$ implies

$$
\tilde{f}_{x}(\gamma \cdot \tilde{y})=\Theta_{f, x}(\gamma) \cdot \tilde{f}_{x}(\tilde{y})=\Theta_{f, x}^{\prime}(\gamma) \cdot \tilde{f}_{x}(\tilde{y})
$$


for all $\tilde{y} \in \tilde{U}_{x}$ and $\gamma \in \Gamma_{x}$. Thus

$$
\left[\Theta_{f, x}^{\prime}(\gamma)^{-1} \Theta_{f, x}(\gamma)\right] \cdot \tilde{f}_{x}(\tilde{y})=\tilde{f}_{x}(\tilde{y}) .
$$

Openness of the embedding implies that there exists $\tilde{y}$ such that $\tilde{f}_{x}(\tilde{y})$ is not a singular point of $\tilde{V}_{f(x)}$. This implies that $\Theta_{f, x}^{\prime}(\gamma)^{-1} \Theta_{f, x}(\gamma)=e$ since $\Gamma_{f(x)}$ acts effectively, whence $\Theta_{f, x}=\Theta_{f, x}^{\prime}$ and $\star f=\star f^{\prime}$.

\section{Proof of Theorem 1 and Corollary 2}

Proof of part (1) For each $x \in X_{\mathcal{P}}$, let $\left(\tilde{U}_{x}, \Gamma_{x, \mathcal{O}}, \rho_{x}, \phi_{x}\right)$ be an orbifold chart for $\mathcal{O}$ about $x$. Let $\tilde{V}_{x} \subset \tilde{U}_{x}, \Lambda_{x, \mathcal{O}}, \Omega_{x, \mathcal{O}}, \psi_{x}$, and $\Gamma_{x, \mathcal{P}}$ be as in the definition of suborbifold. Denote by $\tilde{i}_{x}: \tilde{V}_{x} \hookrightarrow \tilde{U}_{x}$ the inclusion map.

Let $q_{x}: \tilde{U}_{x} / \Lambda_{x, \mathcal{O}} \rightarrow \tilde{U}_{x} / \Gamma_{x, \mathcal{O}}$ be the natural quotient map and define

$$
\phi_{x}^{\prime}=\phi_{x} \circ q_{x} \circ \tilde{i}_{x} / \Lambda_{x, \mathcal{O}}: \tilde{V}_{x} / \Lambda_{x, \mathcal{O}} \rightarrow U_{x}
$$

as in this commutative diagram:

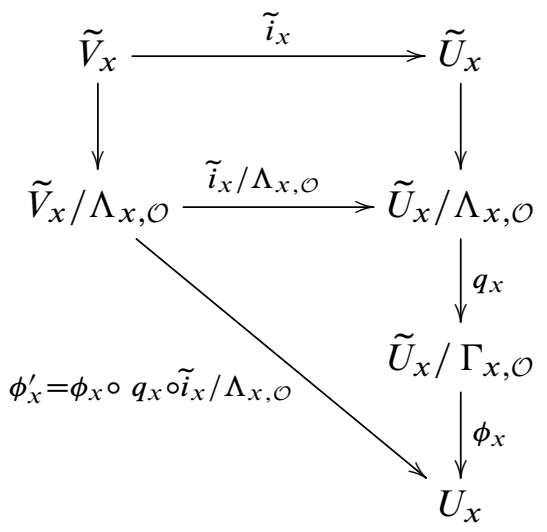

For $\tilde{y} \in \tilde{V}_{x}$, let $\Lambda_{x, \mathcal{O}}(\tilde{y})=\left\{\gamma \in \Gamma_{x, \mathcal{O}}: \gamma \cdot \tilde{y} \in \tilde{V}_{x}\right\}$. Then

$$
\Lambda_{x, \mathcal{O}} \subset \bigcap_{\tilde{y} \in \tilde{V}_{x}} \Lambda_{x, \mathcal{O}}(\tilde{y}) .
$$

By definition, $\mathcal{P}$ is saturated if and only if $\Lambda_{x, \mathcal{O}} \cdot \tilde{y}=\Lambda_{x, \mathcal{O}}(\tilde{y}) \cdot \tilde{y}$ for all $\tilde{y} \in \tilde{V}_{x}$. We claim that $\phi_{x}^{\prime}$ is a homeomorphism onto its image if and only if $\mathcal{P}$ is saturated. To see this, note that $\mathcal{P}$ is not saturated if and only if there exists $x \in X_{\mathcal{P}}$ and $\delta \in \Gamma_{x, \mathcal{O}}$ so that for some $\tilde{y} \in \widetilde{V}_{x}, \delta \cdot \tilde{y} \in \widetilde{V}_{x}$, but $\delta \cdot \tilde{y} \neq \lambda \cdot \tilde{y}$ for any $\lambda \in \Lambda_{x, \mathcal{O}}$. Thus, $\tilde{z}=\delta \cdot \tilde{y}$ satisfies $\Lambda_{x, \mathcal{O}} \cdot \widetilde{z} \neq \Lambda_{x, \mathcal{O}} \cdot \tilde{y}$. Since $\phi_{x}^{\prime}\left(\Lambda_{x, \mathcal{O}} \cdot \widetilde{z}\right)=\phi_{x}^{\prime}\left(\Lambda_{x, \mathcal{O}} \cdot \tilde{y}\right)$, because $\tilde{y}$ and $\tilde{z}$ are 
in the same orbit under the full group $\Gamma_{x, \mathcal{O}}$, we see that $\phi_{x}^{\prime}$ is not a homeomorphism. Thus, we have shown that if $\phi_{x}^{\prime}$ is a homeomorphism, then $\mathcal{P}$ is saturated. To show that $\mathcal{P}$ is saturated implies $\phi_{x}^{\prime}$ is a homeomorphism, note that $\phi_{x}^{\prime}$ is clearly continuous, and since $\Gamma_{x, \mathcal{O}}$ is finite, $\phi_{x}^{\prime}$ is open. As shown above, $\mathcal{P}$ saturated implies that $\phi_{x}^{\prime}$ is injective.

Now, since $\Omega_{x, \mathcal{O}}$ fixes $\tilde{V}_{x}$ by definition, there is a natural identification $I: \tilde{V}_{x} / \Lambda_{x, \mathcal{O}} \leftrightarrow$ $\tilde{V}_{x} /\left(\Lambda_{x, \mathcal{O}} / \Omega_{x, \mathcal{O}}\right)=\tilde{V}_{x} / \Gamma_{x, \mathcal{P}}$ and we have the following diagram:

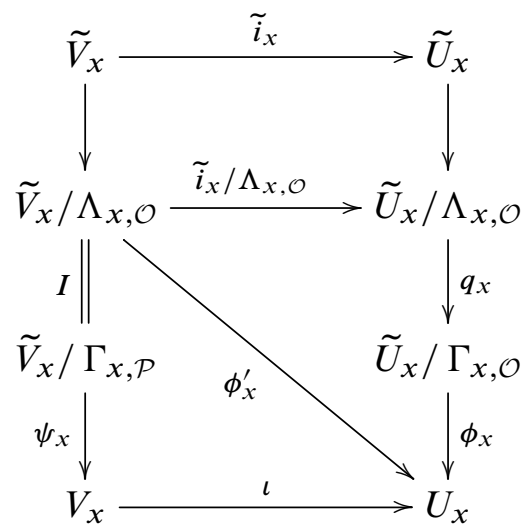

Let $\mathcal{P}^{\prime}$ be the orbifold defined by the local charts $\left(\tilde{V}_{x}, \Gamma_{x, \mathcal{P}}, \rho_{x}^{\prime}, \psi_{x}\right)$, where $\rho_{x}^{\prime}$ is the induced action of $\Gamma_{x, \mathcal{P}}$ on $\widetilde{V}_{x}$ by restricting $\rho_{x}$ to $\Lambda_{x, \mathcal{O}}$ and the action to $\widetilde{V}_{x}$. The required topological embedding $\iota: X_{\mathcal{P}^{\prime}} \rightarrow X_{\mathcal{O}}$ with $\iota\left(X_{\mathcal{P}^{\prime}}\right)=X_{\mathcal{P}}$ is given in local charts by: $\iota=\phi_{x}^{\prime} \circ I^{-1} \circ \psi_{x}^{-1}: V_{x} \rightarrow U_{x}$ and is covered by the inclusion maps $\widetilde{i}_{x}$. This completes the proof of part (1).

Proof of part (2) Let $\sigma_{x, \mathcal{P}}$ be a splitting of the exact sequence

$$
1 \longrightarrow \Omega_{x, \mathcal{O}} \longrightarrow \Lambda_{x, \mathcal{O}} \stackrel{\iota_{x, \mathcal{O}}}{\longrightarrow} \Gamma_{x, \mathcal{O}} \frac{q}{\underset{\sigma_{x, \mathcal{P}}}{\gtrless} \Lambda_{x, \mathcal{O}} / \Omega_{x, \mathcal{O}}=} \Gamma_{x, \mathcal{P}} \longrightarrow 1 .
$$

Let $\Theta_{\iota, x}=\iota_{x, \mathcal{O}} \circ \sigma_{x, \mathcal{P}}: \Gamma_{x, \mathcal{P}} \rightarrow \Gamma_{x, \mathcal{O}}$, where $\iota_{x, \mathcal{O}}=\Lambda_{x, \mathcal{O}} \hookrightarrow \Gamma_{x, \mathcal{O}}$ is the inclusion map. $\Theta_{\iota, x}$ is clearly an injective homomorphism and note that the existence of $\Theta_{\iota, x}$ is equivalent to the existence of $\sigma_{x, \mathcal{P}}$. Let $\tilde{i}_{x}: \widetilde{V}_{x} \hookrightarrow \widetilde{U}_{x}$ be the inclusion map. It is easy to see that $\tilde{i}_{x}$ is $\Theta_{\iota, x}$-equivariant: Let $\tilde{y} \in \tilde{V}_{x}$ and let $\gamma \in \Gamma_{x, \mathcal{P}}$. Then $\gamma \cdot \tilde{y}=\tilde{i}_{x}(\gamma \cdot \tilde{y})$. On the other hand, $\Theta_{\iota, x}(\gamma)=\gamma \omega$ for some $\omega \in \Omega_{x, \mathcal{O}}$ since $q \circ \Theta_{\iota, x}=$ Id. Thus, $\Theta_{\iota, x}(\gamma) \cdot \tilde{y}=\gamma \omega \cdot \tilde{y}=\gamma \cdot \tilde{y}$, since $\omega \cdot \tilde{y}=\tilde{y}$. Thus, ${ }_{\star} \iota=\left(\iota,\left\{\tilde{i}_{x}\right\},\left\{\Theta_{\iota, x}\right\}\right): \mathcal{P}^{\prime} \rightarrow \mathcal{P} \subset \mathcal{O}$ is a complete orbifold embedding that covers the topological embedding $\iota: X_{\mathcal{P}^{\prime}} \rightarrow X_{\mathcal{P}} \subset X_{\mathcal{O}}$. This completes the proof of part (2). 
Proof of Corollary $2 X$ is the point set of a length-minimizing curve in $\mathcal{O}$, so there exists a unit-speed parametrization $c:[a, b] \rightarrow \mathcal{O}$ such that $c([a, b])=X$. We show that $X$ is the underlying space of a suborbifold $\mathcal{P} \subset \mathcal{O}$ that is saturated and split and thus is the image of an orbifold embedding by Theorem 1. Fix $t \in[a, b]$, and let $x=c(t)$. It follows from the characterization of length-minimizing geodesic segments in [2, Theorem 3, page 32] or [3, Proposition 15] that $c$ is contained in the closure of single connected open stratum of $\mathcal{O}$. That is, $\left.c\right|_{(a, b)}$ lies in a subspace of $\mathcal{O}$ with constant isotropy. This implies that $X$ has the structure of a suborbifold $\mathcal{P} \subset \mathcal{O}$. These results also imply that $\Gamma_{x, \mathcal{P}}=\{e\}$, and thus $\mathcal{P}$ has a trivial orbifold structure and so $\mathcal{P}$ is split. Let $\tilde{c}_{x}$ be a lift of $c$ to $\tilde{U}_{x}$. Since $\Gamma_{x, \mathcal{P}}=\{e\}$, we have $\Lambda_{x, \mathcal{O}} \cdot \tilde{y}=\tilde{y}$ for all $\tilde{y} \in \tilde{c}_{x}$. If $\mathcal{P}$ were not saturated at $x$, then there exists $s<s^{\prime} \in[a, b]$ and $\gamma \in \Gamma_{x, \mathcal{O}}$ with $\gamma \cdot \tilde{c}_{x}(s)=\tilde{c}_{x}\left(s^{\prime}\right)$. This implies that $c$ contains a loop in $\mathcal{O}$. This contradicts the property that a length-minimizing curve $c$ must minimize length between any of its points. This contradiction implies $\mathcal{P}$ is saturated and thus, by Theorem $1, \mathcal{P}$ is the image of a complete orbifold embedding. This completes the proof of Corollary 2.

\section{Table of examples and suborbifold properties}

Here is a summary table of properties of suborbifolds possessed by the examples presented in this article which show that all possible combinations of properties that are not implied by others can occur:

\begin{tabular}{l|ccccc} 
& suborbifold & full & saturated & split & image of orbifold embedding \\
\hline Example 8 & yes & yes & yes & yes & yes \\
Example 9 & yes & yes & yes & yes & yes \\
Example 10 & yes & no & yes & yes & yes \\
Example 11 & yes & no & yes & yes & yes \\
Example 12 & yes & yes & yes & no & no \\
Example 13 & yes & no & no & yes & no \\
Example 14 & yes & no & yes & no & no \\
Example 15 & no & - & - & - & no
\end{tabular}

Acknowledgment We are grateful to the referee and wish to thank him for his useful comments and valuable corrections that lead to improvement of the final manuscript.

\section{References}

[1] A Adem, J Leida, Y Ruan, Orbifolds and stringy topology, Cambridge Tracts in Mathematics 171, Cambridge Univ. Press (2007) MR2359514 
[2] J E Borzellino, Riemannian geometry of orbifolds, $\mathrm{PhD}$ thesis, University of California, Los Angeles (1992) MR2687544 Available at http://search.proquest.com/ docview/304009974

[3] J E Borzellino, Orbifolds of maximal diameter, Indiana Univ. Math. J. 42 (1993) 37-53 MR1218706

[4] J E Borzellino, V Brunsden, Orbifold homeomorphism and diffeomorphism groups, from: "Infinite dimensional Lie groups in geometry and representation theory", (A Banyaga, J A Leslie, T Robart, editors), World Scientific, River Edge, NJ (2002) 116137 MR1926425

[5] J E Borzellino, V Brunsden, A manifold structure for the group of orbifold diffeomorphisms of a smooth orbifold, J. Lie Theory 18 (2008) 979-1007 MR2523149

[6] J E Borzellino, V Brunsden, Elementary orbifold differential topology, Topology Appl. 159 (2012) 3583-3589 MR2973378

[7] J E Borzellino, V Brunsden, The stratified structure of spaces of smooth orbifold mappings, Commun. Contemp. Math. 15 (2013) 1350018, 37 MR3117354

[8] W Chen, Y Ruan, Orbifold Gromov-Witten theory, from: "Orbifolds in mathematics and physics”, (A Adem, J Morava, Y Ruan, editors), Contemp. Math. 310, Amer. Math. Soc. (2002) 25-85 MR1950941

[9] W Chen, Y Ruan, A new cohomology theory of orbifold, Comm. Math. Phys. 248 (2004) 1-31 MR2104605

[10] C-H Cho, H Hong, H-S Shin, On orbifold embeddings, J. Korean Math. Soc. 50 (2013) 1369-1400 MR3126596

[11] S Choi, Geometric structures on 2-orbifolds: Exploration of discrete symmetry, MSJ Memoirs 27, Math. Soc. Japan, Tokyo (2012) MR2962023

[12] M W Hirsch, Differential topology, Graduate Texts in Mathematics 33, Springer, New York (1976) MR0448362

[13] M Kankaanrinta, A subanalytic triangulation theorem for real analytic orbifolds, Topology Appl. 159 (2012) 1489-1496 MR2879379

[14] E Lerman, Orbifolds as stacks?, Enseign. Math. 56 (2010) 315-363 MR2778793

[15] I Moerdijk, D A Pronk, Orbifolds, sheaves and groupoids, K-Theory 12 (1997) 3-21 MR1466622

[16] AD Pohl, The category of reduced orbifolds in local charts (2015) arXiv: 1001.0668v5

[17] I Satake, On a generalization of the notion of manifold, Proc. Nat. Acad. Sci. USA 42 (1956) 359-363 MR0079769

[18] I Satake, The Gauss-Bonnet theorem for V-manifolds, J. Math. Soc. Japan 9 (1957) 464-492 MR0095520 
[19] W P Thurston, The geometry and topology of three-manifolds, lecture notes, Princeton University (1979) Available at http://msri.org/publications/books/gt3m

Department of Mathematics, California Polytechnic State University

1 Grand Avenue, San Luis Obispo, CA 93407, USA

Department of Mathematics and Statistics, Penn State Altoona 3000 Ivyside Park, Altoona, PA 16601, USA

jborzell@calpoly.edu, vwb2@psu.edu

Received: 17 June 2014 Revised: 2 December 2014 
